Gazi University
Journal of Science
http://dergipark.gov.tr/gujs

\title{
Current and Future Trend Opportunities of Thermoelectric Generator Applications in Waste Heat Recovery
}

\author{
Mohammad Ruhul Amin BHUIYAN ${ }^{1}$, , Hayati MAMUR ${ }^{2, *}$ (D) , Mehmet Ali USTUNER ${ }^{2}$, Omer Faruk \\ DILMAC $^{3}$ (D) \\ ${ }^{1}$ Islamic University, Department of Electrical and Electronic Engineering, 7003, Kushtia, Bangladesh \\ ${ }^{2}$ Manisa Celal Bayar University, Department of Electrical and Electronics Engineering, 45140, Manisa, Turkey \\ ${ }^{3}$ Çankirl Karatekin University, Department of Chemical Engineering, 18100, Çankirl, Turkey
}

Highlights

- TEG applications were investigated.

- TEGs are widely used for thermal energy harvesting in low power applications.

- Increasing ZT value will promote the application areas.

- Advances in nanotechnology are promising for TEGs.

- The use of TEG is very important for sustainable energy power requirements.

\begin{tabular}{l} 
Article Info \\
\hline $\begin{array}{l}\text { Received: 08 May 2021 } \\
\text { Accepted: 28 Aug } 2021\end{array}$ \\
Keywords \\
\hline Thermoelectric generator \\
Waste heat \\
Energy harvesting \\
Seebeck effect \\
Sustainable energy
\end{tabular}

\section{INTRODUCTION}

Worldwide in the present century, the researchers are highly concerned with increasing the production of thermal to electrical energy in order to develop the industry [1], transport [2], communication [3], buildings [4], and automotive applications [5]. The recent energy crisis can solve by managing electrical energy in a better way to increase thermoelectric generator (TEG) efficiency. The need for developing research in the alternative energy source has driven the drastic climate change in the world. The rapid changes in climates are mainly attributing to utilize fossil fuels for energy generation and transport sectors. Many countries over the world schedules to minimize primary consumption of energy through an increase in alternative energy generation [6], distribution [7], decline $\mathrm{CO}_{2}$ emissions due to climatic challenges, and advance the application of TEGs [8]. TEG applications deal with the recovery of a certain part of the waste heat as electrical energy. Thus, they contribute to increasing energy efficiency and reducing greenhouse gas emissions. [9]. They are more convenient to compare the characteristics of specimens as appear in the expression of the efficiency and play a crucial role in electrical energy developing purpose [10]. They are 
also used for cooling purposes, low heat recovery management, and power in the battery to charge. The techniques of supplying more power to a battery system depending on a temperature difference between the thermoelectric module (TEM) surfaces can easily use in TEGs [11]. Figure 1(a) illustrates the role of TEGs in waste heat sources. The main barrier of TEGs is low efficiency and high cost for advancement the common applications. It is a challenge for the development of TEGs. Some manufacturers have tried to search for cost-effective TE material compositions acting to minimize the effects of low productivity. The forming of TEGs considers a process approach to optimize the whole system because the TEMs have low performance [12]. An elaborated presentation of TEG applications is essential for different challenges that confines the industrial fulfilment of TEGs. This review gives a way that shows current and future trend opportunities for thermoelectric generator applications in waste heat recovery for TEGs utilizations. Waste thermal energy is abundantly energy that each working machine and moving things such as electronic integrated circuits, mobile communications, computer devices, vehicles, power plants, natural gas fields, industries, and even in the human body realizes to the environment as waste heat [13, 14]. TEGs are active devices for converting the thermal into electrical energy. They are widely used in various sectors that become a zone of interest in the field of energy recovery systems from small $[15,16]$ to large types of utilizations, depending on size, generated power, and formed materials [17, 18]. Figure 1(b) shows a block diagram of waste heat recovery.

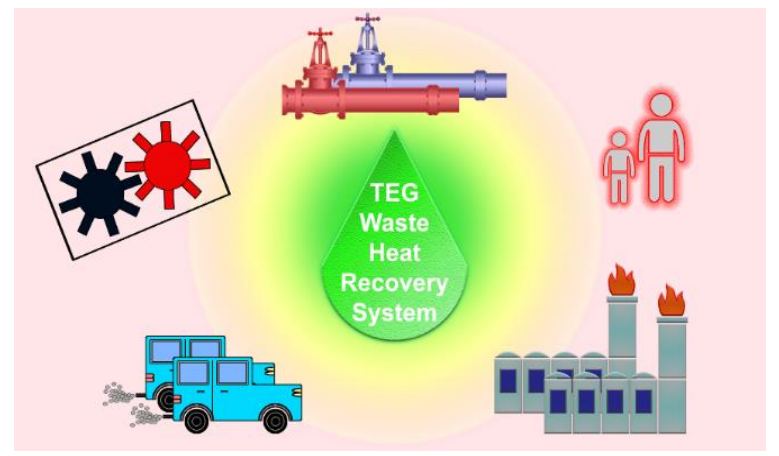

(a): Waste heat sources

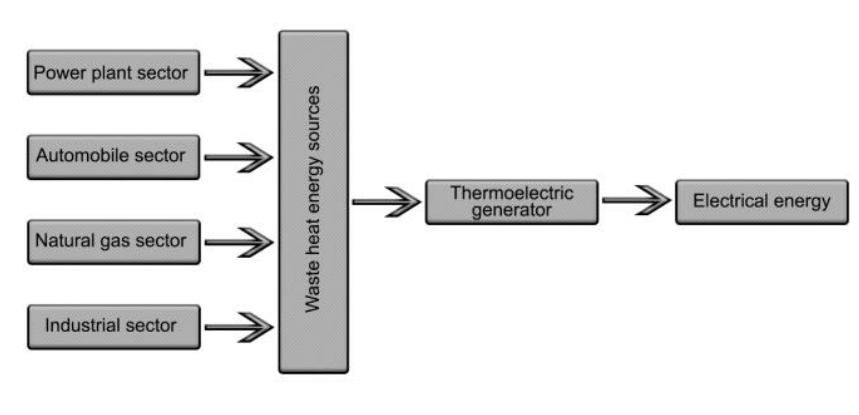

(b): Waste heat recovery block diagram

Figure 1. The role and energy conversion of TEGs in waste heat sources

The waste heat recovery system is an important subject to technological developments. It is in this context that TEGs are slowly developing that consist of a set of TEMs placed between a cold and a hot heat exchanger. The heat exchangers can amplify heat transfer from the heat source to the module to form powerful TEG. The TEMs are connected in series for electrical and in parallel for thermal achievements. They directly convert the heat passes through them into electrical energy. The converters that are an electrical device are regulate and transfer the electric energy to a defined voltage level corresponding to the battery groups and capacitors according to the needs of the end user [19]. The ratio of electrical energy stored or supplied to the end user for the consuming power of this system is known as the overall efficiency. A power consumed is received on the hot heat sink that includes the mechanical power required to function the process by cooling of cold side and pressure losses in the heat exchangers [20]. The researchers deal with the availability of the different charge carrier TE materials with a high figure of merit (ZT) to expanding more applications of TEG. The preparation of omics contacts between metallic interconnects and thermoelements with maximum heat transfer management is also essential [21, 22]. In this context, heat exchangers, coupling with TE modules, electrical converters, and the electrical connection of TEMs were improved in [23]. TEG applications are favorable to researchers because it can provide power from micro sensors to the internet of things (IoT). On the other hand, organic TEGs trying to be newly developed are still undergoing research in the research laboratory [24]. These have been a new scientific interest research area as an alternative research topic to conventional inorganic TEGs during the last decades [25]. One of the recommended materials for organic TEGs is the conductive polyaniline [26]. This conductive polymer material is inexpensive, has a wide range of production and unmatched mechanical flexibility [27]. Although these can be used for low waste heat recovery such as body waste heat, they are not suitable at high temperatures [28]. Their operating temperatures can go up to $250^{\circ} \mathrm{C}$. Some studies on these are 
ongoing. In the future, the widespread use of these will increase and they will appear in the markets [29]. The exclusive presentation of TEG applications in remote environments, micro power generation in biomedical applications, misuse heat management in the automobile, solar TE power in generators, gaining electrical energy of small appliances in homes far from the electricity grid have been presented by means of the paper in the developing countries. They have new properties that operating range, cost, weight, and non-toxicity can open the prospect for the large-scale industrial development of TEG applications. In author's earlier reports [30-36], it is noted that $\mathrm{Bi}_{2} \mathrm{Te}_{3}$ and $\mathrm{Bi}_{2} \mathrm{Te}_{3}$-based nanostructured materials are more suitable for TEG device manufacture in TE applications. Furthermore, in author's previous reports [3743], it is showed that nanostructured materials have been more acceptable for device manufacturing compared to thin films. There are some situations that limit the use of TEGs. The search for new material to increase the ZT values of the materials used in TEGs continues at the level of nano materials. There are also efforts to increase operating temperatures in applications where these materials are used. In addition, engineers and researchers continue to work to increase low efficiency and reduce installation costs to reduce the restrictions in their applications. TEGs can meet the small power requirements of some devices. In this regard, Amatya and Ram [44] conducted a study expressing solar TEG applications in 2010. Ahiska and Mamur [45] published a study on the use of TEGs in renewable energy in 2014. After a while, Champier conducted a study summarizing the new developments and applications in TEG applications in 2017 [46]. Pourkiaei et al. [47] conducted a study describing the potential applications of TEGs and thermoelectric coolers (TECs).

Recent advances in technology and nanoscience have been continuing at a dazzling rate. These advances are also related to the TEGs. To follow the developments, this study makes a general survey of the studies conducted in the last five years. For this reason, this review paper explains the present interest for applications of TEGs. Moreover, the paper will discuss the extensive state of the art of the applications of TEGs and inspect the future prospect. After making a general introduction in the presentation of this study, low power and high-power energy generation applications of TEGs are given in the second chapter. In the third part, the reasons limiting the use of TEGs and the difficulties that need to be overcome are mentioned, and in the fourth part, a future perspective is drawn by giving general results.

\section{REVIEW ON APPLICATION OF THERMOELECTRIC GENERATORS}

Thermoelectric technology is one of the green energy source converters that can provide electrical energy to electric and electronic systems. The waste heat energy source from ambient sources is converted into electrical energy. The TEG technology exploits the Seebeck effect. This effect explains the transformation of heat gradient to electric energy at the different carrier junctions of the TE specimens of a TEG module. The produced electrical energy is highly reliable and a robust energy form, which targets to recover waste heat energy. It is used for different applications such as medical instruments, sensors, and consumer electronics. Waste heat recovery has developed in context the produce electrical energy by using the TEGs that are currently promising research in the world. It consists of a set of TEMs inserted between hot and cold heat exchangers. Every TEM has composed of several pairs of thermoelements connected with thermally in parallel and electrically in series. The modules directly convert a part of the waste thermal energy, which can pass through them into electrical energy. TEG applications can be classified two categories such as large and micro power applications as electrical energy power generation. The first classified TEGs have millimetric dimensions. These TEGs, which are electrically and thermally connected to each other to increase power, find application in high power applications. When looking at high power applications, it is seen that a certain part of the waste heat is for recovery in processes where waste heat is intense. The second classified TEGs utilize at low waste heat. They generate electrical power from $\mu \mathrm{W}$ to a few $\mathrm{mW}$. For this reason, they are used for small power requirements. These are performed based on a TE heat equilibrium approach. It is very significant and mostly is utilized for industrial advancement. The zones or applications, in which waste heat sources are released, play an important role in determining the usage areas of TEGs. According to their utilizations, it is possible to divide the application areas of TEGs into five sections. These are as follows. 1) They are used to meet the electrical energy needs of devices that require less power in areas where there is no electricity network [48]. 2) They are highly preferred for the recovery of waste heat released by burning fossil fuels [49]. Thus, they contribute to energy efficiency. 3) They are used in decentralized domestic energy and thermal energy production processes where sustainable 
energy sources are widely used [50]. 4) They can be preferred to meet the energy needs of small electronic sensors and devices that require little energy in environments with thermal waste heat [51]. 5) These TEGs can also find a place in the production of electrical energy by using the heat of the sun in electrical energy production facilities from solar energy. In fact, hybrid applications are encountered with these solar systems [52].

\subsection{High-power Applications}

The thermal based energy on the TE effect is the most used harvesting for thermal into electrical energy. The obtaining energy mainly relates to the manufacture of TEG devices. An interesting material for flexible TE applications is solution-phase exfoliated graphene. However, the traditional oxidative route has many difficulties of poor flake standard and a lack of quality doping procedure to obtain different charge carriers complementary. T.G. Novak et al. [53] could achieve both extremely high electrical conductivity and high Seebeck coefficients. Their results show the remarkable power factors $\sim 655 \mu \mathrm{W} / \mathrm{mK}$ at room temperature. Silicon nanowires have emerged as a more promising TE specimen owing to a low thermal and a high electric conductance. A high-power TEG manufacture by using a large collection of heavily p-doped silicon nanowires was developed in [54]. The area of several $\mathrm{mm}^{2}$ of macroscopic TEG collects with TE features of silicon, which has a high-power value of $1.8 \mathrm{~W} / \mathrm{mK}$ and low thermal conductivity. Seebeck coefficient, thermal conductivity, and maximum power output of the manufactured devices were discussed. The power density of the manufactured TEG was over a few $\mathrm{mW} / \mathrm{cm}^{2}$ at temperature differences over $100^{\circ} \mathrm{C}$. Other benefit of the used silicon was that the power factor raised with the temperature. One of the important points here is to achieve low thermal conductivity. In this circumstance, the decrease of the thermal conductivity was associated to the roughness spring from the plasma etching process. With attention to other processes, these also show that large quantities of nanowires can be produced without expensive high-resolution lithography. The manufactured materials with decreased thermal conductivity will be very useful for practical applications, as it will allow high temperature ranges to be obtained. When all these are evaluated together, there remains a parameter that needs to be improved. This is the electrical resistance. It is very important to consider the Seebeck constant, measured resistance and thermal resistance values to improve the electrical resistance. Some automotive manufacturers are exploring to convert the waste heat energy of exhaust gas into electric energy. For this reason, modelling and analysis of a TEG are important. W. He et al. [55] examined in detail how changes in the exhaust temperature would provide a change in the generated maximum power value that could be obtained. They have optimized the exhaust exchanger to achieve the best waste heat dispersion on TEG surfaces.

The interest of the aviation industry and space companies in wireless structural health monitoring (SHM) processes is increasing day by day. Thanks to the fact that TEGs convert the temperature difference between their surfaces directly into electrical energy and can obtain wide temperature differences in the said sectors, the use of these devices in these areas for energy generation is of interest. A great number of wires and piezoelectric elements have required to developing the SHM process into aircraft decoration. It is of great importance that the devices placed in these processes take up optimum space and use the areas they cover effectively. Considering that they use these areas for energy recovery, there will be gain after gain. The design procedure of a piezoelectric sensor network with shared signal transmission cables was performed in [56]. High power applications are performed through industrial electronic devices, automobile engines, and aerospace, etc. These applications require different supplying power and dimensions. The TEGs used for these applications have millimeter dimensions and give the output power several Watts at high temperature gradients. These types of TEGs can usually use for industrial purposes.

Industrial sectors release high temperatures that thermal energy into the environment, which know as waste heat energy. This waste heat amount is very high and increases the greenhouse gas effect in the environment. One of the ways to prevent this from being thrown into the environment is to use TEG devices to recover this energy to a certain extent [57]. Moreover, the industrial heat energy management process has one of the economic opportunities for any industry sector. The industrial organic cycles, thermodynamic cycles, burners, and boiler technologies can transform this heat into electricity through TEGs. A stone wool manufacturing plant to convert waste heat into electrical energy from the hot gas flow was considered in [58]. 
Waste heat is a complex problem in integrated circuits and other industrial electronic devices. Industrial different units develop a huge amount of thermal energy as waste heat, depending on its product type. The waste heat energy dissipated from the various sections while operating these processed operations could be turned into an advantage for recycling this as thermal to electrical energy by using TEGs to supply other parts in the enabled electronic devices. Microelectronic TEGs can recover misuse heat into electrical energy of integrated circuits. The silicon integrated circuit can perform by chip thermal management of energy sources for different sensors. G. Hu et al. [59] reported the silicon integrated circuit TEGs that had a highpower generation rate. According to the authors report, TEGs created on industrial silicon complementary metal-oxide-semiconductor (CMOS) process terminal by fabricating nanostructure silicon thermopiles could generate high power about $29 \mu \mathrm{W} / \mathrm{cm}^{2} \mathrm{~K}^{2}$ at near room temperature. Silicon base TEGs conventionally employee long. They are the nanowires of about 10 to $100 \mathrm{~nm}$, which suspended on a cavity to cut-off the bypass of the heat current and secured the temperature difference across its. Moreover, the cavity structure increased the fabrication cost and weakened the mechanical strength of the device manufacture. M. Tomita [60] developed a cost-effective high-power density TEG for portable and wearable objects charge. The relevance of electrical energy harvesting is clean energy that depends only on the recycle of waste heat energy. These waste heat energies from industrial processes and other devices are given into the atmosphere without being exploited any effective device, which is harmful to human and any lives [61].

The limited supply of electrical power generation on waste heat energy recovery in the industrial sector by using TEG systems become a crucial topic day by day. The use of industrial waste heat energy in lighting is among the purposes of TEGs. The low energy requirements of the energy-efficient lighting bulbs that have been introduced recently make the use of TEGs in this area widespread. Dan Dai et al. [62] setup a liquid metal-based TEG system. They developed a prototype study that combines the electromagnetic pump, which is easily available in the market, with TEGs. Ahiska and Mamur [63] applied on a LED bulb.

Management of excessive heat found in industries is also a challenge and must be tackled on its own. Engineers and academics are still working on this subject. Moreover, the production of sufficient number of technological devices to recycle these high waste heat energies is at the commercial stage of operation. The increasing energy need in the industry has increased the electricity used and has begun to reflect on these bills. Increasing awareness of climate change in the world requires addressing this high-energy waste heat energy. Different electricity generation technologies extracted from industrial excess heat at lowtemperature conditions were proposed in [64]. Industrial excess heat management technology is one of these. The technology can evaluate to the temperature condition of heat source, capacity, efficient, and economical aspect. This technology can use the heat of different temperature ranges by TEG devices. Temperatures up to $1300^{\circ} \mathrm{C}$ is required for the hot formation process of steel in the industry. When the environment brought to these temperature values is released into the air after use, it cools and disappears and causes the environment to heat. The use of TEGs to utilize this waste heat can have a positive effect to a certain extent. Thus, the energy efficiency of the system will be increased. In addition, the electrical energy generated from such systems can even be given to the electricity grid. A system for TEG heat management from stationary industrial processes was fulfilled [65].

The reduction of $\mathrm{CO}_{2}$ and waste heat emitted by vehicles using petroleum and its derivatives is one of the difficult issues that are being studied and solved today. In a vehicle operating with an internal combustion engine, two thirds of the fuel are emitted as waste heat energy. A large amount of this is taking place from the exhaust. The fact that the exhaust temperature is very high compared to the ambient temperature is promising in achieving the temperature difference required for TEGs, which increases the output power linearly. For this reason, it is quite common to use TEGs in the exhaust outlets. A holistic TEG for automotive vehicle applications was developed in [66]. X. Liu et al. [67] constructed a waste heat recovery system that extracts temperature from an automotive exhaust pipe and convert the thermal heat energy into electrical energy by using the constructed TEG system. Their result shows a promising potential performance of using this kind of TEG for low-temperature misuse heat recovery vehicles. Y. Zhang et al. [68] generated a high-power density of $5.26 \mathrm{~W} / \mathrm{cm}^{2}$ with the temperature difference between the surfaces of TEGs at $500^{\circ} \mathrm{C}$. 
Recently, the reduction in energy consumption, greenhouse gas emissions, and environmental pollutions has faced more challenges in automotive industries in the implementation of the TEG system. The TEGs can enable a direct conversion of thermal energy into electrical energy without any moving parts and maintenance by employing the exhaust heat management process. The electrical energy supplies are especially helpful to give the producing electrification trend in transport moving vehicles. A performance of a temperature-controlled TEG with embedded heat pipes concept in a light duty vehicle was tested in [69]. It showed that the greenhouse gases emission was reduced. Today, very good progress has been made in vehicle fuel systems to minimize carbon emissions. With the developing technology, the carbon emissions of vehicles have also been minimized. In addition, increasing the use of low carbon emission fuels in vehicles has also contributed to this. In addition, the greenhouse gas effect can be reduced with the use of external combustion engines. These motors are preferred for electric energy trains. Hybrid vehicles using electric drive and petrol-fueled engines are available in new technologies. W.B. Nader et al. [70] emphasized the importance of increasing the quality factor of the TEG system to achieve the performance obtained in internal combustion engines. T.Y. Kim et al. [71] obtained maximum output power around 118 $\mathrm{W}$ and the energy conversion efficiency around $2.1 \%$ by using twelve TEGs under the engine operating conditions. The authors also investigated the energy production performance of hexagonal-shaped TEG waste heat recovery system for passenger vehicle applications [72]. Waste heat management processes can increase power recycle to diminish fossil fuel consumption, the harmful impact of $\mathrm{CO}_{2}$ and global warming. Although the efficiency of TEGs in the recovery of waste heat energy as electrical energy is low, they are used as reliable and maintenance-free energy recovery devices. J. Wang et al. [73] focused on the performance of TEGs under different running conditions within different positions. They found that TEG positions were very effective in output power. Y. Choi et al. [74] have fabricated thirty TEGs system for a waste heat management of diesel engines by using porous medium-assisted generator equipped with customized a TEG.

Most of the fuel energy is rejected to become waste heat in every motor vehicle. The TEGs can possess high potential performance by recover this heat to enhance fuel efficiency. M. Aljaghtham et al. [75] investigated a high thermal gradient is creating in air cooling at the bottom surface and hot oil at the top surface of TEG for the TEG conversion. They optimized TEG numbers and measures in their work. In the optimization process, they considered different oil pan geometries and driving conditions. Then, they carried out simulation studies according to these optimization values. For multi-stage oil pan geometry optimization, they obtained the temperature difference between the surfaces of the TEGs of $76^{\circ} \mathrm{C}$ and in this case, they found an output power density of about $5.77 \mathrm{~kW} / \mathrm{m}^{2}$. In the end, they argued that the developed TEG system was an applicable waste heat recovery methodology for combustion engines and could improve fuel performance. The output power can be achieved by TEGs around $700 \mathrm{~W}$, with air inlet temperatures up to $620^{\circ} \mathrm{C}$ [76]. During road tests, a manufactured TEG system has supplied an output power achievement of at least $450 \mathrm{~W}$ in some motor vehicles. Furthermore, the TEG system achieved same electric power output with repeatable performance in over six months of vehicle testing in bench and engine dynamometer testing.

The aerospace power sources are the complex thermodynamic systems, which consists of multiple subsystems such as aerodynamics, mechanical, heat and mass transfer, thermal, and other forms of interactions [77]. The used electrical energy in aerospace technology depends on various processes. The required electricity can generate in different ways in these technologies. One of them is the TEGs that can easily produce electrical energy in aerospace technology. The flight test instrumentation, maintenance, cabin crew support, and structural health monitoring (SHM) system are the most common utilization fields for energy harvesting in aerospace. The promising facilities of aerospace agencies in the wireless SHM process will advance the TEG research of efficient power sources and energy harvesting. Every aircraft connected a TEG system can produce electrical energy from environmental energy source with temperature gradient at various locations. The temperature difference between the surfaces of TEGs can be increased by combining thermal diffusion receivers with TEG energy conversion systems. This temperature difference is the value between ambient temperature and outdoor temperature. The most common heat sink used in this area are fins with a flat surface as a heat sink. In these, the structure of each fins can be easily modified. Among the most important features sought in heat expanders for these applications are their light weight and small. Researchers can easily design heat expanders of the same weight and height with the 
development of nanotechnology. S. Boccardi et al. [78] carried out the enhancement of TEG efficiency by manufacturing cooled air heat sink for SHM applications at the low power wireless systems. Their work investigated design, manufacture, and testing of the proposed thermal diffusion process. Their goal was to increase the temperature difference between TEG surfaces. The experimental results provided higher temperature differences in the proposed thermal diffusion process. Therefore, traditional cylindrical pin-fin heat sinks can be compared with higher output power. Eventually, their results illustrated that the heat sink of the TEG arrangement can be able to generate electrical power. The electric power backup of the onboard power distribution line and the zero-power wireless sensor is a suitable power supply in aerospace applications. The SHM process could be the utilization of energy harvesting devices. Recently, the SHM systems are becoming a real possibility of energy harvesting in aerospace environments. The wireless sensor nodes are the most promising system that can able to communicate wirelessly to a central base station and make decisions on the damage. The process points to the existence of both active and passive monitoring techniques to detect damage to structures. The vibration and thermal gradients are the two energy sources that could be developed for aircraft electrical energy purposes. Furthermore, piezoelectric transducers mounted to the surface of a structure can utilize to produce electrical power. The viability of two energy sources such as vibration and thermal gradients studied in [79]. In the study, the vibration range and temperature difference were $20-400 \mathrm{~Hz}$ and $50^{\circ} \mathrm{C}$, respectively. These energy harvesting processes revealed a great potential in powering current wireless SHM systems. An electrical power up to $1 \mathrm{~mW}$ could be generated for a single vibrational energy recovery system. The numerical modelling advancement the optimizing of orientation and position of the vibrational harvester high levels of energy could be achieved. Over the flight period, it generated average power levels between 5 and $30 \mathrm{~mW}$ by using the commercial TEGs. The energy harvesting system show great potential in powering into wireless SHM systems, whereas power requirements was range between 1 and $180 \mathrm{~mW}$. Finally, it recommended that the most promising process would be utilized in wireless sensor nodes that can be able to communicate this wirelessly to a central base station.

Non-uniform mechanical and thermal loading and vibrations can be induced unintended stresses. The edge of TEG dislocations has occurred with confirms the failure due to shear stresses caused by heat expansion. Plastic deterioration on the surfaces is not seen. Also, in many cases, a traceable pattern does not occur on their broken surfaces. The TEG elements have developed to both thermal and mechanical loading. Clamping is the main structure of this production for mechanical loading. Thermal loads due to vibrations can cause malfunctions. This happens when the stress threshold value of the device is exceeded. Axial stresses are more effective for vertically placed elements. When bending and cutting occur in the areas where the device placements are located, device malfunctions are very likely. Therefore, the orientation and shape of TEG devices are of great importance in minimizing thermal and structural loads. In this context, a topology optimization approach is proposed by researchers to minimize both thermal and structural stresses on TEG. J. Mativo et al. [80] developed the compliant TEGs in aerospace applications by using topology optimization for multifunctional materials. L. Janak et al. [81] developed a backup electric power source based on the micro electromechanical system (MEMS) with TEM. The aerospace sector is under the continuous advancement of the technology used for performance and effectiveness. Recently, the main goal is related to the creation of a cost-effective and environmentally friendly aircraft system. These goals can be easily achieved using TEMs. S.A. Sánchez et al. [82] proposed a magnetic field in a thermocouple that improved the performance of the systems.

\subsection{Low-power Applications}

Day by day, the objects in our daily lives are becoming smarter. They are connected to the Internet. These devices can now be controlled via the Internet. These objects created an IoT-based society. The use of portable and wearable TEGs can be expanded to establish Internet connections. TEG waste heat recovery systems can be used some low power areas like medical, wearable devices, IoTs, and wireless sensor networks. With the advances in flexible and organic TEG technologies and nano technologies, these areas of use are expected to increase even more. When these applications are taken into consideration, the use of TEGs of different sizes and various strengths is encountered here. Thanks to the low energy consumption of some devices, the use of these organic and flexible TEGs at low power is gradually increasing. Almost all applications using TEGs require little power. The primary energy source of systems of grid off is the 
battery. It is an advantage that TEGs are available for the energy source of this battery to be sustainable. Nevertheless, Although TEGs provide energy for decades in autonomous systems, the battery used has a certain lifespan. It must be replaced at the end of its life. As can be understood from here, although TEG systems do not require maintenance for a long time, the tools combining the autonomous energy system have maintenance requirements.

The wearable devices can play a crucial role to keep a constant monitor on vitals in the hospital. Doctors can be used wearable devices to measure the vitals without the patient being restricted to bed. Health monitoring wearable devices powered by TEGs can also monitor how much the patient walks and how many calories the patient has consumed. With the development of technology and nanomaterials, it is predicted that the applications of these devices will increase even more. The data generated by wearable devices either provide information to their own processor or to a laptop, and these are collected for later analysis. The devices that monitor the blood pressure of the patients are among the most used of these devices. Thanks to these devices, continuous communication between the patient and the doctor can be established. The waste heat energy released by man depends on his physical activities. M. Thielen et al. [83] performed an optimization study on how much of the human body waste heat can be recycled. In their studies, they reached $260-280 \mu \mathrm{W}$ electrical energy output power and $13-14 \mu \mathrm{W} / \mathrm{cm}^{2}$ power density with a flexible TEG system placed on the human wrist. Figure 2(a) shows a power regulation block diagram and Figure 2(b) presents the process of an electrical energy recovery system from waste heat energy with TEG from a wearable device.

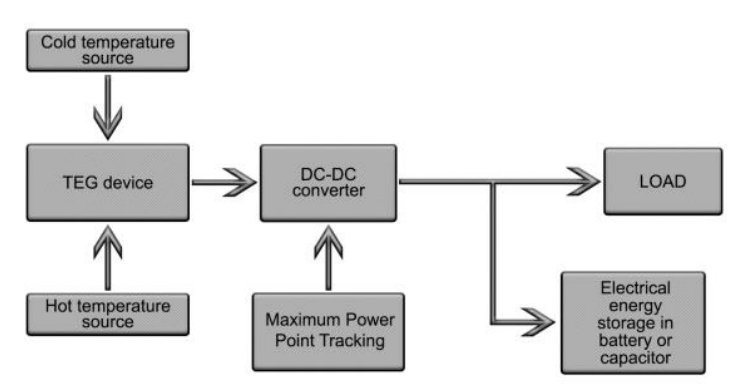

(a): The power regulation block diagram

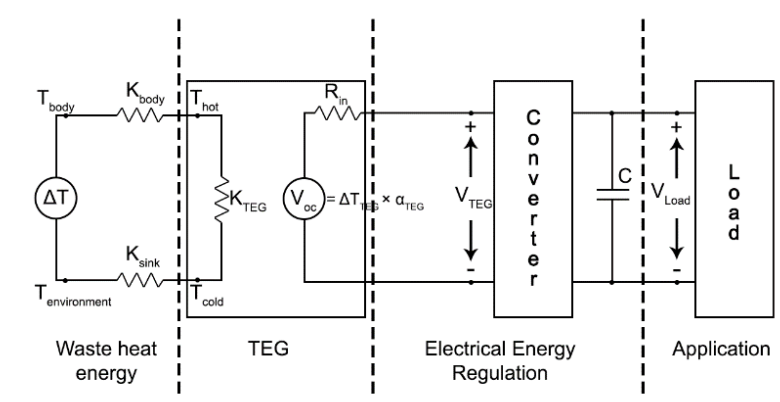

(b): The conversion system from body heat

Figure 2. The processes of an electrical energy recovery system of TEGs

The primary purpose of wearable TEG devices is to provide uninterrupted energy needs of electronic components used without batteries by using the temperature difference between the waste heat of the human body and the ambient temperature [84]. Different methods have been proposed by the researchers to use the waste heat of the human body. In fact, the devices using such wearable devices have started to appear in the markets [85]. A wearable electrocardiography [86], a miniaturized accelerometer [87], a self-powered wearable pressure sensing system [88] and a wireless sensor system [89] were reported on a wearable TEG using the human body heat as the energy source for self-powered wearable devices for low power applications. Among these are self-powered TEG systems developed for electrocardiogram (ECG) [86]. ECG provides information about the state of the heart. It requires little energy to operate its electronics. A self-powered ECG device that gets its energy from TEG is built on a flexible circuit board. TEG derives its energy from the temperature difference between body temperature and ambient temperature. Due to the nature of TEGs, this device has been capable of meeting the energy needs for decades without requiring maintenance. In the healthcare system, self-powered devices powered by organic and flexible TEGs can be used for motion detection [87]. The device contains more than 50 pairs of cubic-shaped TE legs of $\mathrm{p}$ - and n-type $\mathrm{Bi}_{2} \mathrm{Te}_{3}$-based materials to obtain electrical energy from the human body temperature. The TEG can be generated a voltage in the millivolt range at different temperatures of $50 \mathrm{~K}$, with quite a low internal resistance. For this circumstance is attached to the arm or a more comfortable body area so that the TEG can absorb waste heat from the human body. The flexible-portable pressure sensors with highly sensitive and cost-effective attributes great demands in artificial intelligence, wearable electronic devices, monitoring heartbeat, biomedical, and blood oxygen saturation. The mobile operation, flexible, ultrathin, 
and sustainable power sources are highly desirable to the researcher for portable pressure sensors. Recently, the researchers developed a self-powered wearable pressure sensing system with a flexible thin film TEG to obtain the human body heat [88]. These devices provide great advantages for constantly monitoring human movements. In case of a dangerous situation, there are alarms to inform the relevant person. The designs of these devices are formed to be attached to human skin and to be supply with TEGs. Wearable devices can be placed in different parts of the human body. These areas include wrist, arm, leg, upper arm, and chest. In a study using TEGs of the same structure, the highest power value was obtained in the wrist with approximately $20 \mu \mathrm{W} / \mathrm{cm}^{2}$ at an air velocity of $1.4 \mathrm{~m} / \mathrm{s}$ according to body region locations [90]. As the air velocity increased, the temperature difference between TEG surfaces increased and the obtained electrical energy power density increased. Medical and wearable sensor systems such as a temperature sensor, pulse oximeter sensor, accelerometer sensor, Bluetooth low energy module, and microcontroller unit are the prominent tools in biomedical applications. These wearable devices are important for the treatment of the disease in the early clinic. They are also preferred for monitoring the patient's body condition.

The TE effect converts the temperature change of material into electrical voltage and vice versa. The best TE material conducts electricity well and offers good thermal insulation. A TE converter that the heat supplied is converted into electricity by using the Seebeck effect. The current thermodynamic efficiency of the materials today available is around $15 \%$ of the Carnot limit. It is the difficulty of decoupling from electrical to thermal conduction. The progress of nanomaterial synthesis techniques and the development of new compositions offer opportunities for the development of a generation of TE materials more efficient, cost-effective, and more flexible for various applications such as biomedical devices. The basic component of a TE device comprises a single structure of semiconductor TE material of type $p$ and $n$, each electrically connected in series. Networking of the electrical devices within electronics embedded can communicate and sense interactions to each other by using the IoT. In future generations, IoT-based thermoelectricity will offer advanced levels of service and practically motivated the way to lead people's daily lives. Thermoelectricity seems to be a candidate for IoT that magnitude of heat losses to direct and reliable conversion. There are two trends to improve in materials integrates, such as pre-existing nanostructure modulation and conventional TE materials. Thermoelectricity-based IoT connections need a low power energy.

Recently developed devices require very small power requirements. Their threshold values can be provided at small voltages. When idle, their power requirements are virtually non-existent. Advancements are in the medical, power, agriculture sectors, and smart cities by using IoT-based thermoelectricity. The development of mobile technologies and the increasing interest in remote sensing systems keep the need for TEGs on the agenda to meet their energy needs [91]. Energy consumption and the need to replace or recharge the battery remain a key challenge for the adoption of IoT, particularly for commercial and industrial applications [92]. Many low-powers portable IoT devices can work forever, powered by energy harvesting, without the need to replace the battery or recharge it. Some of the devices can operate without batteries in remote areas by the low power energy harvesting. The combination of energy harvesting processes and low-power design can create IoT applications with connectivity, processing, and tracking functions. Over 40 billion devices are currently connected to the internet. In the future, expect this number will rise to 50 billion [93]. The main components used in IoT devices are embedded systems, cloud computing system and big data systems, and internet connection systems [94, 95]. In a study by M. Haras et al. [96], an innovative miniaturize pulse amplifier for the TEG energy acquisition system was proposed. The TEG system proposed by them was operated intermittently periodically with the heat source. The recommend boosting process was in a structure that could be form with IoT nodes. As a result of their work, they stated that TEG produces higher power than traditional operation. They also suggested that this exhibited performance be observed with low temperature differences for IoT devices. The authors' development was based on optimization of TEG's operating conditions rather than the generator itself. Ultimately, they argued that their approach to using TEG for battery or resource would bring cheaper and reliable alternatives and IoT device expansion. a characterization of several power management units that focused on wearable healthcare IoT devices that was supplied by a TEG energy conversion system was presented in [97]. 
TEGs are devices that meet the electrical energy needs of both devices that need communication with each other to a certain extent. For this reason, it has started to attract a great deal of attention in IoT systems. The usability of TEGs for alternate power sources in the wireless sensor network was showed in [98]. Th study illustrated that TEGs meet the power requirements of low-power sensing nodes and outperform batteries as a cost-effective setup, successfully. Thermal energy in the form of metabolic heat is a promising source for robust supplying a power to electronic components. The IoT with integrated wireless sensor networks become important issues that increasing demand for renewable power supply and management. H. Park et al. [99] have demonstrated the application of TEG energy harvesting system as a renewable energy source for IoT, including wireless sensor networks. They investigated the models of the human thermoregulatory process on body heat harvesting. Finally, the authors addressed a combination with a DC-DC converter to boost its low output voltage for power management. A simulation-based analysis for defining the performance of TEC with the power management integrated circuit for harvesting heat energy was given in [100].

Wireless sensor networks (WSNs) are networks that detect physical and chemical changes in different processes or regions with the help of sensors and combine them at one point to evaluate them, operate independently from each other, and have wireless connectivity. A WSN consists of hundreds or even thousands of sensors drop linked together. Developments in electronics have enabled WSN networks to communicate with each other with low cost and low power requirement. Thanks to their ability to work with low electrical energy, TEGs have had a wide range of uses. In addition, WSNs have found a wide range of use thanks to their wireless communication. Due to these features, it has become a communication network that can be preferred in the military field. Moreover, the human physiological data, tracking, and monitoring can be done by doctors inside a hospital through a WSN [101-105]. Every technical process produces waste heat. Modern wireless sensor modules require only $\sim 100 \mu \mathrm{W}$ to $10 \mathrm{~mW}$. The electrical power requirements of WSNs range from $100 \mu \mathrm{W}$ to $10 \mathrm{~mW}$. The decrease in this value is due to the development of new technology electronics. Considering the electrical energy and lifetime produced by TEGs, these are quite suitable for WSNs. A transient modelling of TEGs for utilization in WSRs nodes as an electric power generator that convert thermal energy into electrical energy was showed in [106]. A TEG system consisting of a double printed circuit that will convert environmental temperature changes into electrical energy to meet the electrical energy needs of WSNs was designed [107].

Today, it has become more popular to supply WSN nodes with thermal energy harvesting TEGs instead of being powered by batteries. Various processes are used to convert the ambient temperature source into electrical energy. Recently, researchers and engineers are moving towards the conversion of waste heat energy, which is abundant in energy processes and environment, into electrical energy. When the thermal energy in the environment is used, electrical energy in mW levels can be obtained by using TEGs. This will increase when the ZT values of TEGs are further increased. A study combining TEGs with WSN nodes was conducted in [108]. The system has been implemented a self-optimization process to deliver the electrical energy obtained from TEG to the WSN node in a continuous and stable manner. J.P Im et al. [109] installed a self-powered WSN operation with silicon-based 3D TEG system for operating temperature sensor, humidity sensor, radio frequency module and integrated circuit. Eventually, they transmitted the data of the humidity and temperature sensors with a radio frequency module and proved the accuracy of this data. A thermoelectric harvester with WSN node on water pipelines at the low-temperature gradient was fulfilled in [110].

\subsection{Solar TEGs}

Integration of TEGs in solar photovoltaic thermal hybrid panels can generate electrical energy. It increases the efficiency of photovoltaic panels. In addition, this hybrid structure results in the production of heat energy. The electrical energy can be consumed in local loads, stored in batteries, and injected into the electronic device network. The heat can be used as hot water, air conditioning, heat pumps, and cooling machines produce absorption in the cooling system [111]. E.A. Chavez-Urbiola et al. [112] investigated the possibility of using TEGs in solar hybrid systems. The authors examined different systems such as radiation concentration, traditional thermal geometry, and TEG heat extractor. They studied experimentally on traditional semiconductor material designed at $\sim 200^{\circ} \mathrm{C}$ temperature. The efficiency of TEG's has almost 
linear dependence on the temperature difference. Over the load power generated of $3 \mathrm{~W}$. The current and voltage were also linear with temperature. In conclusion, the relation to the efficiency as well as some recommendations is to optimal solar cells for applications in these systems. A.H. Yavuz et al. [113] proposed and analyzed the solar TEG assisted a water pump for agricultural irrigation purposes. Authors designed a single module solar TEG cell with average solar radiation in the Turkey region and calculated its electrical parameters. Authors used a tar-coated, heat-absorbing copper plate, $94^{\circ} \mathrm{C}$ the hot surface temperature, and $21^{\circ} \mathrm{C}$ the cold surface temperature to developing the system. The simulated a solar TEG panel corresponded to a $275 \mathrm{~W}$ photovoltaic panel. Finally, authors obtained the $187 \mathrm{~W} / \mathrm{m}^{2}$ of power, whereas solar TEG panel can provide $1000 \mathrm{~W} / \mathrm{m}^{2}$. S. Su et al. [114] investigated the high-efficiency solar TEGs with non-homogeneously doped nanomaterials. Authors composed a solar TEG within a flat-panel collector without the optical concentration in a vacuum enclosure. They designed quantum-confined electrons in thermoelectric nanostructure materials to achieved reversible electron transport. After that, they calculated the maximum efficiency is much larger compare to the previous report. The performance revealed that the influences of the current density and thermal conductivity. Photovoltaic modules with TEGs are directly converted into electric power from solar radiation. The process is known as a solarpowered hybrid power source. TEG module has a small output power with the temperature difference between its two surfaces. For this circumstance, individually, TEGs cannot be utilized in solar heat harvesting. Overcome this problem required a prototype of the proposed photovoltaic-thermoelectric hybrid power source. Some researcher solves this defect through modelling, simulation, design, performance evaluation, electrical and mechanical investigation [115-119]. The contributions of these research results can be outlined under as follows: 1) The hybrid power source can directly convert both solar radiation and heat into electric energy. 2) It can enable utilizing the TEGs for low-grade thermal energy harvesting. 3) It provides a constant voltage, so other power generation systems can be added by connecting them through a converter. 4) The system uses a high-efficient maximum power point tracking with the adaptive and variable step size.

The power values obtained from the analyzed applications have been presented in Table. It should be noted that the temperature difference between the hot and cold surfaces of TEGs, ZT, the number of TEGs used and the Seebeck constants of the TEGs affect the amount of generated power from these TEGs. Since the amount of waste heat emitted from the exhaust of automotive is high, the number of TEGs used has been increased, and as a result, the amount of power produced has increased. The generated power could be used in places with higher energy requirements. Fewer TEGs are needed for feeding electronic devices with low power requirements. As a result, the generated power values are less. These TEGs are used in the fields of IoT, wireless sensors and biosensors.

\section{LIMITATION AND CHALLENGE}

The TEGs are low efficient performance due to the lower ZT compare to other energy conversion technologies. The human body and atmosphere temperature difference low, sufficient thermal energy is not provided as input to TEG, so sometimes it does not work properly. It does not allow additional practical heat sinks due to small size and flexibility for cooling purposes. The internal and external load of TEG is the other difficulty to harvest optimum energy. Its efficiencies are very limited due to their thermal and electrical behavior being dependent on each other. Recently, thermoelectric nanostructure material technology makes them more efficient in some selective applications purpose. However, the TEGs implementation in IoT devices would help advance applications. The approaches of these should be overcome by the following criteria: 1) It can easily harvest energy in dark, hidden locations where the only available energy sources are heat losses. 2) Because they are partially commercialized devices, they do not have a standardized power cost. 3) It is reliable, silent, requires low or no maintenance and is easily scalable. 4) High-quality, cost-effective, and tunable TE properties. 5) Compacted for machining or 
Table. The power values obtained from the current applications analyzed

\begin{tabular}{|l|l|l|l|l|l|l|l|}
\hline $\begin{array}{l}\text { Application } \\
\text { Area }\end{array}$ & $\begin{array}{l}\text { Number } \\
\text { of TEGs }\end{array}$ & $\begin{array}{l}\text { Generated } \\
\text { Power }\end{array}$ & Ref. & $\begin{array}{l}\text { Application } \\
\text { Area }\end{array}$ & $\begin{array}{l}\text { Number } \\
\text { of TEGs }\end{array}$ & $\begin{array}{l}\text { Generated } \\
\text { Power }\end{array}$ & Ref. \\
\hline Automotive & 64 & $1538 \mathrm{~W}$ & {$[69]$} & Solar & 1 & $85.5 \mathrm{~mW}$ & {$[117]$} \\
\hline Automotive & 224 & $1392 \mathrm{~W}$ & {$[5]$} & Roadway & 4 & $47.14 \mathrm{~mW}$ & {$[2]$} \\
\hline Automotive & 400 & $1000 \mathrm{~W}$ & {$[68]$} & Wireless & 1 & $34 \mathrm{~mW}$ & {$[109]$} \\
\hline Automotive & 60 & $944 \mathrm{~W}$ & {$[67]$} & Aerospace & 1 & $30 \mathrm{~mW}$ & {$[78]$} \\
\hline Automotive & 32 & $751 \mathrm{~W}$ & {$[75]$} & Aerospace & 1 & $25 \mathrm{~mW}$ & {$[77]$} \\
\hline Automotive & 18 & $627 \mathrm{~W}$ & {$[73]$} & IoT & 1 & $7.6 \mathrm{~mW}$ & {$[106]$} \\
\hline Process & 50 & $388 \mathrm{~W}$ & {$[65]$} & Waste heat & 2 & $7 \mathrm{~mW}$ & {$[11]$} \\
\hline Solar & 87 & $275 \mathrm{~W}$ & {$[112]$} & Wireless & 1 & $2 \mathrm{~mW}$ & {$[110]$} \\
\hline Waste heat & 40 & $240 \mathrm{~W}$ & {$[62]$} & IoT & 1 & $1.719 \mathrm{~mW}$ & {$[100]$} \\
\hline Automotive & 12 & $118 \mathrm{~W}$ & {$[71]$} & Environment & 1 & $1.48 \mathrm{~mW}$ & {$[107]$} \\
\hline Automotive & 18 & $98.8 \mathrm{~W}$ & {$[72]$} & IoT & 1 & $400 \mu \mathrm{W}$ & {$[96]$} \\
\hline Automotive & 30 & $98.3 \mathrm{~W}$ & {$[74]$} & Human body & 1 & $260 \mu \mathrm{W}$ & {$[83]$} \\
\hline Waste heat & 20 & $41 \mathrm{~W}$ & {$[63]$} & Sensors & 1 & $229 \mu \mathrm{W}$ & {$[99]$} \\
\hline Waste heat & 20 & $29 \mathrm{~W}$ & {$[61]$} & Accelerometer & 1 & $192.6 \mu \mathrm{W}$ & {$[87]$} \\
\hline Solar & 7 & $15 \mathrm{~W}$ & {$[115]$} & Body heat & 1 & $156.5 \mu \mathrm{W}$ & {$[16]$} \\
\hline Solar & 19 & $14 \mathrm{~W}$ & {$[111]$} & Micropower & 1 & $100 \mu \mathrm{W}$ & {$[54]$} \\
\hline Waste heat & 30 & $11.5 \mathrm{~W}$ & {$[1]$} & Human body & 1 & $38 \mu \mathrm{W}$ & {$[86]$} \\
\hline Micropower & 1 & $1.8 \mathrm{~W}$ & {$[44]$} & Micropower & 1 & $29 \mu \mathrm{W}$ & {$[59]$} \\
\hline IoT & 1 & $0.53 \mathrm{~W}$ & {$[3]$} & Body heat & 1 & $18.58 \mu \mathrm{W}$ & {$[51]$} \\
\hline Solar PV & 1 & $0.5 \mathrm{~W}$ & {$[20]$} & Micropower & 1 & $10 \mu \mathrm{W}$ & {$[60]$} \\
\hline Wireless & 1 & $115.7 \mathrm{~mW}$ & {$[108]$} & Waste heat & 1 & $0.4 \mu \mathrm{W}$ & {$[12]$} \\
\hline
\end{tabular}

device-controllable behavior. 6) Compacted nanoscale features. 7) Enhanced the ZT value over the bulk materials. 8) High thermal stability for extended with time. 9) High TE efficiency. Continued research on this field to gain a more quantitative understanding is necessary to allow the applications. This research field will optimize nanostructure thermoelectric semiconductor materials and accelerate the wide adoption of thermoelectric technologies in different applications.

\section{CONCLUSION AND FUTURE RESEARCH}

Although the efficiency of TEGs is low and their installation costs are high, their usage areas are expanding thanks to their long-life operation under extreme conditions without requiring maintenance, and their ability to harvest electrical energy from thermal energy. In this review paper, the TEG applications for different purposes have been presented. These applications required high costs for installations. Therefore, it has a limited sector. Moreover, TEGs have proven their extreme reliability through these applications. The application of TEGs in various sectors is within certain perspectives. Some of the researchers achieved success in various TEG applications. Interest in these applications has revived with the advent of modern technology that makes it possible to overcome the critical hurdle of TEG applications. In terms of efficiency and environment, current solutions are focused on the development of TEG applications with efficient nanostructured thermoelectric materials with interconnected electrical and thermal properties of these materials, and their new designs can allow for better integration into energy conversion systems.

\section{ACKNOWLEDGEMENT}

The authors are grateful to the Department of Electrical and Electronic Engineering, Islamic University for providing some facilities.

\section{CONFLICTS OF INTEREST}

No conflict of interest was declared by the authors. 


\section{REFERENCES}

[1] Quan, R., Li, T., Yue, Y., Chang, Y., and Tan, B., "Experimental study on a TE generator for industrial waste heat recovery based on a hexagonal heat exchanger", Energies, 13(12): 3137, (2020).

[2] Tahami, S. A., Gholikhani, M., and Dessouky, S., "TE energy harvesting system for roadway sustainability", Transportation Research Record, 2674(2): 135-145, (2020).

[3] Saraereh, O. A., Alsaraira, A., Khan, I., and Choi, B. J., "A hybrid energy harvesting design for onbody Internet-of-Things (IoT) networks", Sensors, 20(2): 407, (2020).

[4] Reddick, C., Sorin, M., Bonhivers, J. C., and Laperle, D., "Waste heat and renewable energy integration in buildings", Energy and Buildings, 211: 109803, (2020).

[5] He, M., Wang, E., Zhang, Y., Zhang, W., Zhang, F., and Zhao, C., "Performance analysis of a multilayer TE generator for exhaust heat recovery of a heavy-duty diesel engine", Applied Energy, 274: 115298, (2020).

[6] Leonard, M. D., Michaelides, E. E., and Michaelides, D. N., "Energy storage needs for the substitution of fossil fuel power plants with renewable", Renewable Energy, 145: 951-962, (2020).

[7] Kim, H., You, H., Choi, K. S., and Han, S., "A study on interconnecting to the power grid fof new energy using the natural gas pressure", Journal of Electrical Engineering \& Technology, 15(1): 307$314,(2020)$.

[8] Araiz, M., Casi, Á., Catalán, L., Martínez, Á., and Astrain, D., "Prospects of waste-heat recovery from a real industry using TE generators: Economic and power output analysis", Energy Conversion and Management, 205: 112376, (2020).

[9] Hussain, T., Li, X., Danish, M. H., Rehman, M. U., Zhang, J., Li, D., Chen, G., and Tang, G., "Realizing high TE performance in eco-friendly SnTe via synergistic resonance levels, band convergence and endotaxial nanostructuring with Cu2Te", Nano Energy, 73: 104832, (2020).

[10] Chuang, F. C., Chang, C. L., Chow, Y. N., Shen, H. C., Wu, T. L., Chen, Y. C., Chiang, C. S., Liao, F. L., and Chen, Y. H., "Application of water tank employing smart sensor for thermal-electric energy conversion on vehicles", Sensors and Materials, 32(1): 135-148, (2020).

[11] Ashraf, M., "A Maximum power-point tracking multiple-input thermal energy harvesting module", International Journal of Electronics and Communications, 121: 153231, (2020).

[12] Kang, M., and Yeatman, E. M., "Coupling of piezo-and pyro-electric effects in miniature thermal energy harvesters", Applied Energy, 262: 114496, (2020).

[13] Weng, M., and Geng, C., "Research on computer centralized management system based on thermal energy data acquisition and display", Thermal Science, 24(5): 3299-3307, (2020).

[14] Cong, P., Zhou, J., Li, L., Cao, K., Wei, T., and Li, K., "A Survey of hierarchical energy optimization for mobile edge computing: A perspective from end devices to the cloud", ACM Computing Surveys, 53(2): 38, (2020).

[15] Huang, L., Lin, S., Xu, Z., Zhou, H., Duan, J., Hu, B., and Zhou, J., "Fiber-based energy conversion devices for human-body energy harvesting", Advanced Materials, 32(5): 1902034, (2020). 
[16] Nozariasbmarz, A., Suarez, F., Dycus, J. H., Cabral, M. J., LeBeau, J. M., Öztürk, M. C., and Vashaee, D., "TE generators for wearable body heat harvesting: material and device concurrent optimization", Nano Energy, 67: 104265, (2020).

[17] Yang, S. M., Wang, J. Y., and Chen, M. D., "On the improved performance of TE generators with low dimensional polysilicon-germanium thermocouples by BiCMOS process", Sensors and Actuators A: Physical, 306: 111924, (2020).

[18] Jia, X., and Guo, Q., "Design study of bismuth-telluride-based TE generators based on TE and mechanical performance", Energy, 190: 116226, (2020).

[19] Zhang, X., Yang, B., Yu, T., and Jiang, L., "Dynamic Surrogate Model based optimization for MPPT of centralized thermoelectric generation systems under heterogeneous temperature difference", IEEE Transactions on Energy Conversion, 35(2): 966-976, (2020).

[20] Sabry, M., Lashin, A., and Al Turkestani, M., "Experimental and simulation investigations of CPV/TEG hybrid system", Journal of King Saud University-Science, 33(2): 101321, (2021).

[21] Narjis, A., Liang, C. T., El Aakib, H., Tchenka, A., and Outzourhit, A., "Design optimization for maximized thermoelectric generator performance", Journal of Electronic Materials, 49(1): 306-310, (2020).

[22] Hegde, G. S., Prabhu, A. N., Rao, A., and Chattopadhyay, M. K., "Enhancement in thermoelectric figure of merit of bismuth telluride system due to tin and selenium co-doping", Materials Science in Semiconductor Processing, 127: 105645, (2021).

[23] Yanagisawa, R., Tsujii, N., Takao, M. O. R. I., Ruther, P., Paul, O., and Nomura, M., "Nanostructured planar-type uni-leg Si TE generators", Applied Physics Express, 13(9): 095001, (2020).

[24] Lindorf, M., Mazzio, K. A., Pflaum, J., Nielsch, K., Brütting, W., and Albrecht, M., "Organic-based thermoelectrics", Journal of Materials Chemistry A, 8(1): 7495-7507, (2020).

[25] Zhao, W., Ding, J., Zou, Y., Di, C. A., and Zhu, D., "Chemical doping of organic semiconductors for thermoelectric applications”, Chemical Society Reviews, 49(20): 7210-7228, (2020).

[26] Xu, Q., Qu, S., Ming, C., Qiu, P., Yao, Q., Zhu, C., Wei, T. R., He, J., Shi, X., and Chen, L., "Conformal organic-inorganic semiconductor composites for flexible thermoelectrics", Energy \& Environmental Science, 13(2): 511-518, (2020).

[27] Du, Y., Xu, J., Paul, B., and Eklund, P., "Flexible thermoelectric materials and devices", Applied Materials Today, 12: 366-388, (2018).

[28] Russ, B., Robb, M. J., Brunetti, F. G., Miller, P. L., Perry, E. E., Patel, S. N., Ho, V., Chang, W. B., Urban, J. J., Chabinyc, M. L., Hawker, C. J., and Segalman, A., "Power factor enhancement in solution-processed organic n-type thermoelectrics through molecular design", Advanced Materials, 26(21): 3473-3477, (2014).

[29] Wang, H., and Yu, C., "Organic thermoelectrics: materials preparation, performance optimization, and device integration", Joule, 3(1): 53-80, (2019).

[30] Mamur, H., Dilmac, O. F., and Bhuiyan, M. R. A., "A review on performance evaluation of Bi2Te3based and some other TE nanostructure materials", Current Nanoscience, 17(3): 423-446, (2021). 
[31] Mamur, H., and Bhuiyan, M. R. A., "Characterization of Bi2Te3 nanostructure by using a costeffective chemical solution route", Iranian Journal of Chemistry and Chemical Engineering, 39(3): 23-33, (2020).

[32] Mamur, H., Bhuiyan, M. R. A., Korkmaz, F., and Nil, M., "A review on bismuth telluride (Bi2Te3) nanostructure for TE applications", Renewable \& Sustainable Energy Reviews, 82: 4159-4169, (2018).

[33] Mamur, H., and Bhuiyan, M. R. A., "Bismuth telluride (Bi2Te3) nanostructure for TE applications", International Scientific and Vocational Studies Journal, 3(1): 1-7, (2019).

[34] Mamur, H., Dilmac, O. F., Korucu, H., and Bhuiyan, M. R. A., "Cost-effective chemical solution synthesis of bismuth telluride nanostructure for TE applications", Micro \& Nano Letters, 13(8): 1117-1120, (2018).

[35] Bhuiyan, M. R. A., and Mamur, H., "Review of the bismuth telluride (Bi2Te3) nanoparticle: growth and characterization", International Journal Energy Applications and Technologies, 3(2): 27-31, (2016).

[36] Bhuiyan, M. R. A., and Mamur, H., "Synthesis and characterization of Se doped Bi2Te3 nanocrystalline materials", Karaelmas Science and Engineering Journal, 9(1): 11-21, (2019).

[37] Akter, M., Khan, M. N. I., Mamur, H., and Bhuiyan, M.R.A., "Synthesis and characterisation of CdSe QDs by using a chemical solution route", Micro \& Nano Letters, 15(5): 287-290, (2020).

[38] Bhuiyan, M. R. A., Miah, M. A. H., and Begum, J., "Substrate temperature effect on the structural and optical properties of ZnSe thin films", Journal of Bangladesh Academy Sciences, 36(2): 233240, (2012).

[39] Bhuiyan, M. R. A., Al Azad, M. A., and Hasan, S. M., "Annealing effect on structural and electrical properties of AgGaSe2 thin films", Indian Journal Pure \& Applied Physics, 49: 180-185, (2011).

[40] Bhuiyan, M. R. A., Saha, D. K., and Hasan, S.M., "Structural and electrical properties of polycrystalline AgxGa2-xSe2 $(0.4 \leq x \leq 1.6)$ thin films", Indian Journal Pure \& Applied Physics, 47: 787-792, (2009).

[41] Bhuiyan, M. R. A., Rahman, M. K., and Firoz Hasan, S. M., "Valence-band characterization of AgGaSe2 thin films", Journal of Physics D: Applied Physics, 41(23): 235108, (2008).

[42] Bhuiyan, M. R. A., and Firoz Hasan, S. M., "Optical properties of polycrystalline AgxGa2-xSe2 $(0.4 \leq \mathrm{x} \leq 1.6)$ thin films", Solar Energy Materials and Solar Cells, 91(2-3): 148-152, (2007).

[43] Bhuiyan, M. R. A., and Firoz Hasan, S. M., "Optical absorption characteristics of polycrystalline AgGaSe2 thin films", Journal of Physics D: Applied Physics, 39(23): 4935, (2006).

[44] Amatya, R., and Ram, R. J., "Solar thermoelectric generator for micropower applications", Journal of Electronic Materials, 39(9): 1735-1740, (2010).

[45] Mamur, H., and Ahiska, R., "A review: Thermoelectric generators in renewable energy", International Journal of Renewable Energy Research (IJRER), 4(1): 128-136, (2014).

[46] Champier, D., "Thermoelectric generators: A review of applications", Energy Conversion and Management, 140: 167-181, (2017). 
[47] Pourkiaei, S. M., Ahmadi, M. H., Sadeghzadeh, M., Moosavi, S., Pourfayaz, F., Chen, L., Yazdi, M. A. P., and Kumar, R., "Thermoelectric cooler and thermoelectric generator devices: A review of present and potential applications, modeling and materials", Energy, 186: 115849, (2019).

[48] Huang, Y., Xu, D., Kan, J., and Li, W., "Study on field experiments of forest soil thermoelectric power generation devices", Plos One, 14(8): e0221019, (2019).

[49] Ramírez, R., Gutiérrez, A. S., Eras, J. J. C., Valencia, K., Hernández, B., and Forero, J. D., "Evaluation of the energy recovery potential of thermoelectric generators in diesel engines", Journal of Cleaner Production, 241: 118412, (2019).

[50] Karthick, K., Suresh, S., Joy, G. C., and Dhanuskodi, R., "Experimental investigation of solar reversible power generation in Thermoelectric Generator (TEG) using thermal energy storage", Energy for Sustainable Development, 48: 107-114, (2019).

[51] Wang, Y., Zhu, W., Deng, Y., Fu, B., Zhu, P., Yu, Y., Li, J., and Guo, J., "Self-powered wearable pressure sensing system for continuous healthcare monitoring enabled by flexible thin-film thermoelectric generator", Nano Energy, 73: 104773, (2020).

[52] Li, G., Shittu, S., Ma, X., and Zhao, X., "Comparative analysis of thermoelectric elements optimum geometry between photovoltaic-thermoelectric and solar thermoelectric", Energy, 171: 599-610, (2019).

[53] Novak, T. G., Kim, J., Kim, J., Tiwari, A. P., Shin, H., Song, J. Y., and Jeon, S., "Complementary ntype and p-type graphene films for high power factor TE generators", Advanced Functional Materials, 30(28): 2001760, (2020).

[54] Elyamny, S., Dimaggio, E., Magagna, S., Narducci, D., and Pennelli, G., "High power TE generator based on vertical silicon nanowires", Nano Letters, 20(7): 4748-4753, (2020).

[55] He, W., Wang, S., and Yue, L., "High net power output analysis with changes in exhaust temperature in a TE generator system", Applied Energy, 196: 259-267, (2017).

[56] Wang, Y., Qiu, L., Luo, Y., Ding, R., and Jiang, F., "A piezoelectric sensor network with shared signal transmission wires for structural health monitoring of aircraft smart skin", Mechanical Systems and Signal Processing, 141: 106730, (2020).

[57] Woolley, E., Luo, Y., and Simeone, A., "Industrial waste heat recovery: A systematic approach", Sustainable Energy Technologies and Assessments, 29: 50-59, (2018).

[58] Araiz, M., Casi, Á., Catalán, L., Martínez, Á., and Astrain, D., "Prospects of waste-heat recovery from a real industry using TE generators: Economic and power output analysis", Energy Conversion and Management, 205: 112376, (2020).

[59] Hu, G., Edwards, H., and Lee, M., "Silicon integrated circuit TE generators with a high specific power generation capacity", Nature Electronics, 2(7): 300-306, (2019).

[60] Tomita, M., Oba, S., Himeda, Y., Yamato, R., Shima, K., Kumada, T., Xu, M., Takezawa, H., Mesaki, K., Tsuda, K., Hashimoto, S., Zhan, T., Zhang, H., Kamakura, Y., Suzuki, Y., Inokawa, H., Ikeda, H., Matsukawa, T., Matsuki, T., and Watanabe, T. "10 $\mu \mathrm{W} / \mathrm{cm} 2-$ Class High power density silicon TE energy harvester compatible with CMOS-VLSI technology", IEEE Symposia on VLSI Technology and Circuits, Hawaii, USA, June 21, 93-94, (2018).

[61] Ando Junior, O. H., Calderon, N. H., and De Souza, S. S., "Characterization of a TE generator (TEG) system for waste heat recovery”, Energies, 11(6): 1555, (2018). 
[62] Dai, D., Zhou, Y., and Liu, J., "Liquid metal based TE generation system for waste heat recovery", Renewable Energy, 36(12): 3530-3536, (2011).

[63] Mamur, H., and Ahiska, R., "Application of a DC-DC boost converter with maximum power point tracking for low power thermoelectric generators", Energy Conversion and Management, 97: 265$272,(2015)$

[64] Johansson, M. T., and Söderström, M., "Electricity generation from low-temperature industrial excess heat—an opportunity for the steel industry", Energy Efficiency, 7(2): 203-215, (2014).

[65] Ebling, D. G., Krumm, A., Pfeiffelmann, B., Gottschald, J., Bruchmann, J., Benim, A. C., Adam, M., Labs, R., Herbetz, R. R., and Stunz, A., "Development of a system for TE heat recovery from stationary industrial processes", Journal of Electronic Materials, 45(7): 3433-3439, (2016).

[66] Kober, M., "Holistic development of TE generators for automotive applications", Journal of Electronic Materials, 49(5): 2910-2919, (2020).

[67] Liu, X., Deng, Y. D., Li, Z., and Su, C. Q., "Performance analysis of a waste heat recovery TE generation system for automotive application", Energy Conversion and Management, 90: 121-127, (2015).

[68] Zhang, Y., Cleary, M., Wang, X., Kempf, N., Schoensee, L., Yang, J., Joshi, G., and Meda, L., "Hightemperature and high-power-density nanostructured TE generator for automotive waste heat recovery", Energy Conversion and Management, 105: 946-950, (2015).

[69] Pacheco, N., Brito, F. P., Vieira, R., Martins, J., Barbosa, H., and Goncalves, L. M., "Compact automotive TE generator with embedded heat pipes for thermal control", Energy, 197: 117154, (2020).

[70] Nader, W. B., "TE generator optimization for hybrid electric vehicles", Applied Thermal Engineering, 167: 114761, (2020).

[71] Kim, T.Y., Kwak, J., and Kim, B. W., "Application of compact TE generator to hybrid electric vehicle engine operating under real vehicle operating conditions", Energy Conversion and Management, 201: 112150, (2019).

[72] Kim, T. Y., Kwak, J., and Kim, B. W., "Energy harvesting performance of hexagonal shaped TE generator for passenger vehicle applications: An experimental approach", Energy Conversion and Management, 160: 14-21, (2018).

[73] Wang, J., Song, X., Li, Y., Zhang, C., Zhao, C., and Zhu, L., "Modeling and analysis of TE generators for diesel engine exhaust heat recovery system", Journal of Energy Engineering, 146(2): 04020002, (2020).

[74] Choi, Y., Negash, A., and Kim, T. Y., "Waste heat recovery of diesel engine using porous mediumassisted TE generator equipped with customized TE modules", Energy Conversion and Management, 197: 111902, (2019).

[75] Aljaghthamand, M., and Celik, E., "Design optimization of oil pan TE generator to recover waste heat from internal combustion engines", Energy, 200: 117547, (2020). 
[76] Crane, D., LaGrandeur, J., Jovovic, V., Ranalli, M., Adldinger, M., Poliquin, E., Dean, J., Kossakovski, D., Mazar, B., and Maranville, C., "TEG on-vehicle performance and model validation and what it means for further TEG development", Journal of Electronic Materials, 42(7): 1582-1591, (2013).

[77] Dong, Z., Li, D., Wang, Z., and Sun, M., “A review on exergy analysis of aerospace power systems”, Acta Astronautica, 152: 486-495, (2018).

[78] Boccardi, S., Ciampa, F., and Meo, M., "Design and development of a heatsink for TE power harvesting in aerospace applications", Smart Materials and Structures, 28(10): 105057, (2019).

[79] Pearson, M. R., Eaton, M. J., Pullin, R., Featherston, C. A., and Holford, K. M., "Energy harvesting for aerospace structural health monitoring systems", Journal of Physics: Conference Series, 382(1): 012025, (2012).

[80] Mativo, J., and Hallinan, K., "Development of compliant thermoelectric generators (TEGs) in aerospace applications using topology optimization", Energy Harvesting and Systems, 4(2): 87-105, (2019).

[81] Janak, L., Ancik, Z., Vetiska, J., and Hadas, Z., "Thermoelectric generator based on MEMS module as an electric power backup in aerospace applications", Materials Today: Proceedings, 2(2): 865$870,(2015)$.

[82] Sánchez, S. A., "Finite element analysis of thermoelectric-galvanomagnetic interactions and their aerospace applications", Aerotecnica Missili \& Spazio, 94(2): 133-135, (2015).

[83] Thielen, M., Sigrist, L., Magno, M., Hierold, C., and Benini, L., "Human body heat for powering wearable devices: From thermal energy to application", Energy Conversion and Management, 131: 44-54, (2017).

[84] Nozariasbmarz, A., Collins, H., Dsouza, K., Polash, M. H., Hosseini, M., Hyland, M., Liu, J., Malhotra, A., Ortiz, F. M., Mohaddes, F., Ramesh, V. P., Sargolzaeiaval, Y., Snouwaert, N., Öztürk, M. C., and Vashaee, D., "Review of wearable thermoelectric energy harvesting: From body temperature to electronic systems", Applied Energy, 258: 114069, (2020).

[85] Zhao, L., Li, H., Meng, J., and Li, Z., "The recent advances in self-powered medical information sensors", InfoMat, 2(1): 212-234, (2020).

[86] Kim, C. S., Yang, H. M., Lee, J., Lee, G. S., Choi, H., Kim, Y. J., Lim, S. H., Cho, S. H., and Cho, B. J., "Self-powered wearable electrocardiography using a wearable thermoelectric power generator", ACS Energy Letters, 3(3): 501-507, (2018).

[87] Wang, Y., Shi, Y., Mei, D., and Chen, Z., "Wearable thermoelectric generator to harvest body heat for powering a miniaturized accelerometer", Applied Energy, 215: 690-698, (2018).

[88] Wang, Y., Zhu, W., Deng, Y., Fu, B., Zhu, P., Yu, Y., Li, J., and Guo, J., "Self-powered wearable pressure sensing system for continuous healthcare monitoring enabled by flexible thin-film thermoelectric generator", Nano Energy, 73: 104773, (2020).

[89] Mohsen, S., Zekry, A., Youssef, K., and Abouelatta, M., "A self-powered wearable wireless sensor system powered by a hybrid energy harvester for healthcare applications", Wireless Personal Communication, 116: 3143-3164, (2020).

[90] Hyland, M., Hunter, H., Liu, J., Veety, E., and Vashaee, D., "Wearable thermoelectric generators for human body heat harvesting", Applied Energy, 182: 518-524, (2016). 
[91] Yoon, Y. S., Zo, H., Choi, M., Lee D., and Lee, H. W., "Exploring the dynamic knowledge structure of studies on the Internet of things: Keyword analysis", ETRI Journal, 40(6): 745-758, (2018).

[92] Madakam, S., Lake, V., Lake, V., and Lake, V., "Internet of Things (IoT): A literature review", Journal Computer and Communications, 3(05): 56616, (2015).

[93] Haras, M., and Skotnicki, T., "Thermoelectricity for IoT-A review”, Nano Energy, 54: 461-476, (2018).

[94] Aceto, G., Persico, V., and Pescapé, A., "Industry 4.0 and health: Internet of things, big data, and cloud computing for healthcare 4.0", Journal Industrial Information Integration, 18: 100129, (2020).

[95] Augustin, A., Yi, J., Clausen, T., and Townsley, W. M., "A study of LoRa: long range \& low power networks for the internet of things", Sensors, 16(9): 1466, (2016).

[96] Haras, M., Markiewicz, M., Monfray, S., and Skotnicki, T., "Pulse mode of operation-A new booster of TEG, improving power up to X2.7-to better fit IoT requirements", Nano Energy, 68: 104204, (2020).

[97] Kilani, D., Alhawari, M., Mohammad, B., Saleh, H., Sanduleanu, M., and Ismail, M., "Cascaded power management unit characterization for TEG-based IoT devices in $65 \mathrm{~nm}$ CMOS", Microelectronics Journal, 90: 285-296, (2019).

[98] Narducci, D., "Thermoelectric harvesters and the internet of things: technological and economic drivers", Journal of Physics: Energy, 1(2): 024001, (2019).

[99] Park, H., Lee, D., Park, G., Park, S., Khan, S., Kim, J., and Kim, W., "Energy harvesting using thermoelectricity for IoT (Internet of Things) and E-skin sensors", Journal of Physics: Energy 1(4): 042001, (2019).

[100] Afghan, S. A., and Géza, H., "Modelling and analysis of energy harvesting in internet of things (IoT): Characterization of a thermal energy harvesting circuit for IoT based applications with LTC3108", Energies, 12(20): 3873, (2019).

[101] Buratti, C., Conti, A., Dardari, D., and Verdone, R., "An overview on wireless sensor networks technology and evolution”, Sensors, 9(9): 6869-6896, (2009).

[102] Cheng, C. T., Chi, K. T., and Lau, F. C., "A delay-aware data collection network structure for wireless sensor networks", IEEE Sensors Journals, 11(3): 699-710, (2010).

[103] Yick, J., Mukherjee, B., and Ghosal, D., "Wireless sensor network survey", Computer Networks, 52(12): 2292-2330, (2008).

[104] Sah, D. K., and Amgoth, T., "Renewable energy harvesting schemes in wireless sensor networks: A survey", Information Fusion, 63: 223-247, (2020).

[105] Fu, X., Fortino, G., Pace, P., Aloi, G., and Li, W., "Environment-fusion multipath routing protocol for wireless sensor networks", Information Fusion, 53: 4-19, (2020).

[106] Miloš, M., Aneta, P., Branislav, R., and Zoran, P., "A transient modeling of the thermoelectric generators for application in wireless sensor network nodes", Electronics, 9(6): 1015, (2020). 
[107] Liao, X., Liu, Y. Ren, J., Guan, L., Sang, X., Wang, B., Zhang, H., Wang, Q., and Ma, T., "Investigation of a double-PCM-based thermoelectric energy-harvesting device using temperature fluctuations in an ambient environment", Energy, 202: 117724, (2020).

[108] Hou, L., and Chen, W., "A novel MPPT method for autonomous wireless sensor networks node with thermal energy harvesting", Engineering Research Express, 2(1): 015005, (2020).

[109] Im, J. P., Kim, J. H., Lee, J. W., Woo, J. Y., Im, S. Y., Kim, Y., Eom, Y. S., Choi, W. C., Kim, J. S., and Moon, S. E., "Self-powered autonomous wireless sensor node by using silicon-based 3D thermoelectric energy generator for environmental monitoring application”, Energies, 13(3): 674, (2020).

[110] Lineykin, S., Sitbon, M., and Kuperman, A., "Design and optimization of low-temperature gradient thermoelectric harvester for wireless sensor network node on water pipelines", Applied Energy, 283: 116240, (2020).

[111] Bayod-Rújula, Á. A., Uche, J., Tejero, J. A., Del Amo, A., Martínez-Gracia, A., and Usón, S., "Integration of thermoelectric generators (TEG) in solar PVT panels", Renewable Energy Power Quality Journal, 17: 495-499, (2019).

[112] Chávez-Urbiola, E. A., Vorobiev, Y. V., and Bulat, L. P., "Solar hybrid systems with thermoelectric generators", Solar Energy, 86(1): 369-378, (2012).

[113] Yavuz, A. H., "Solar thermoelectric generator assisted irrigation water pump: Design, simulation and economic analysis", Sustainable Energy Technologies and Assessments, 41: 100786, (2020).

[114] Su, S., and Chen, J., "Simulation investigation of high-efficiency solar thermoelectric generators with inhomogeneously doped nanomaterials", IEEE Transactions on Industrial Electronics, 62(6): 35693575 , (2014).

[115] Fathabadi, H., "Novel solar-powered photovoltaic/thermoelectric hybrid power source", Renewable Energy, 146: 426-434, (2020).

[116] Chen, W. H., Wang, C. C., Hung, C. I., Yang, C. C., and Juang, R. C., "Modeling and simulation for the design of thermal-concentrated solar thermoelectric generator", Energy, 64: 287-297, (2014).

[117] Liu, H. B., Meng, J. H., Wang, X. D., and Chen, W. H., "A new design of solar thermoelectric generator with combination of segmented materials and asymmetrical legs", Energy Conversion and Management, 175: 11-20, (2018).

[118] Abdo, A., Ookawara, S., and Ahmed, M., "Performance evaluation of a new design of concentrator photovoltaic and solar thermoelectric generator hybrid system", Energy Conversion and Management, 195: 1382-1401, (2019).

[119] Shittu, S., Li, G., Xuan, Q., Zhao, X., Ma, X., and Cui, Y., "Electrical and mechanical analysis of a segmented solar thermoelectric generator under non-uniform heat flux", Energy, 199: 117433, (2020). 